Ion Transfer

\title{
Fast Ion-Transfer Processes at Nanoscopic Liquid/Liquid Interfaces**
}

\author{
Qing Li, Shubao Xie, Zhongwei Liang, Xin Meng, Shujuan Liu, Hubert H. Girault,* and \\ Yuanhua Shao*
}

Ion-transfer (IT) reactions at a liquid/liquid (L/L) interface or at an interface between two immiscible electrolyte solutions (ITIES) are of great fundamental interest because they play a key role in many important physicochemical and biological systems, such as in phase-transfer catalysis, sensors, drug delivery, and biological membranes. ${ }^{[1]}$ The kinetics of IT reactions at such interfaces has been the central focus in the past few decades, and is often very fast and difficult to measure. Many electrochemical methods including chronocoulometry, ac impedance, and cyclic voltammetry have been extensively used to probe the kinetics of IT processes. ${ }^{[1]}$ Unfortunately, measurements by these conventional electrochemical methods at macroscopic (e.g., millimeter-sized) ITIES are easily affected by uncompensated ohmic potential drop ( $i R$ drop) and capacitive currents. To minimize those effects, Girault et al. ${ }^{[2]}$ introduced in 1986 a micrometer-sized liquid/liquid ( $\mu$-L/L) interface supported at the tip of a pulledglass micropipette, and in 1997 Mirkin et al. ${ }^{[3]}$ further reduced the liquid/liquid interface to the nanometer range by using nanopipettes. Voltammetry at nanopipettes and scanning electrochemical microscopy are currently the most reliable techniques used to extract the fast kinetic rate constants of ion-transfer reactions at ITIES. ${ }^{[1 e, 4]}$ The fastest rate constants reported so far are in the range of a few $\mathrm{cm} \mathrm{s}^{-1} \cdot{ }^{[3,5-7]}$

Steady-state voltammetry at solid ultramicroelectrodes has been employed extensively to measure the rapid kinetics of heterogeneous electron-transfer reactions, and the methodologies developed are transposable to IT reactions at micrometer-scale or nanometer-scale ITIES (micro- and nano-ITIES, respectively). ${ }^{[1 \mathrm{e}]}$ Assuming that for a thin-wall pipette (e.g., $\mathrm{RG} \leq 2, \mathrm{RG}=r_{\mathrm{g}} / r$, where $r_{\mathrm{g}}$ is the outer radius of the glass insulator and $r$ the inner radius, that is, the radius of

[*] Q. Li, S. Xie, Z. Liang, X. Meng, S. Liu, Prof. Dr. Y. Shao

Beijing National Laboratory for Molecular Sciences

College of Chemistry and Molecular Engineering

Peking University, Beijing 100871 (China)

Fax: (+86) 10-6275-1708

E-mail:yhshao@pku.edu.cn

Homepage: http://www.chem.pku.edu.cn/index.php?id $=196$

Prof. Dr. H. H. Girault

Laboratoire d'Electrochimie Physique et Analytique

Ecole Polytechnique Fédérale de Lausanne

1015 Lausanne (Switzerland)

Fax: $(+41)$ 21-693-3667

E-mail: hubert.girault@epfl.ch

[**] This work was supported by NSFC $(20735001,20628506)$ and the Innovation Team Programs of the Ministry of Education of China. H.H.G. is grateful to Peking University for a visiting professorship.

(1) Supporting information for this article is available on the WWWW under http://dx.doi.org/10.1002/anie.200903143. the interface) the micro- and nano-ITIES are essentially uniformly accessible, and the potential dependence of the rate constant follows the Butler-Volmer equation as corroborated by the excellent agreement between theory and experimental voltammograms, the standard rate constant $\left(k^{0}\right)$, and the charge-transfer coefficient $(\alpha)$, which can be determined by fitting an experimental voltammogram to a theoretical equation. ${ }^{[8]}$ Although several groups have reported kinetic measurements of IT reactions at micro-ITIES, ${ }^{[1 \mathrm{e}]}$ many IT reactions are too fast to be probed with a micro-ITIES under steady-state conditions. The nano-ITIES supported at nanopipettes should be advantageous because the mass-transport rate can be enhanced according to Equation (1), ${ }^{[5]}$ where $m_{0}$ is

$m_{0}=D / r_{\text {app }}$

the mass-transport rate, $D$ the ion diffusion coefficient, and $r_{\text {app }}$ the effective radius. $m_{0}$ is therefore inversely related to interface radius, implying that the maximum measurable value of $k^{0}$ increases linearly with $1 / r_{\text {app }}$. Hence, it is not surprising that faster rate constants can be determined by nanopipette voltammetry.

We report here on our kinetic studies of several IT reactions such as cation transfer (tetraethylammonium, $\mathrm{TEA}^{+}$), anion transfer (perchlorate, $\mathrm{ClO}_{4}^{-}$), and facilitated ion transfer (FIT) of $\mathrm{K}^{+}$by dibenzo[18]crown-6 (DB18C6) at the nanoscopic interface between water and 1,2-dichloroethane (nano-W/DCE) supported at nanopipettes with effective tip radii approximately equal to $1 \mathrm{~nm}$. The two theoretical models developed yield almost the same equation and were used to analyze the observed steady-state cyclic voltammograms.

The configuration of the experimental setup is illustrated in Figure 1. The electrochemical systems for characterization of nanopipettes and studies of IT and FIT can be represented as Cells $\mathrm{A}, \mathrm{B}$, and $\mathrm{C}$, respectively.

$\mathrm{Ag}|\mathrm{AgTPBCl}| 2$ mм DB18C6 + 2 mм BTPPATPBCl (DCE) ||

$100 \mathrm{~mm} \mathrm{KCl}(\mathrm{W})|\mathrm{AgCl}| \mathrm{Ag}$

Cell A

$\mathrm{Ag}|\mathrm{AgTPBCl}| 2$ mм BTPPATPBCl (DCE) ||

$2 \mathrm{~mm} \mathrm{TEACl}+10 \mathrm{~mm} \mathrm{KCl}(\mathrm{W})|\mathrm{AgCl}| \mathrm{Ag}$

Cell B

$\mathrm{Ag}|\mathrm{AgTPBCl}| 2$ mм BTPPATPBCl (DCE) ||

$1 \mathrm{~mm} \mathrm{NaClO}_{4}+10 \mathrm{~mm} \mathrm{LiCl}(\mathrm{W})|\mathrm{AgCl}| \mathrm{Ag}$

Cell C

Here $\mathrm{TPBCl}$ refers to tetrakis(4-chlorophenyl)borate and BTPPATPBCl stands for bis(triphenylphosphoranylidene) ammonium tetrakis(4-chlorophenyl)borate, a lipophilic salt used to provide ionic conductivity to the DCE phase. 


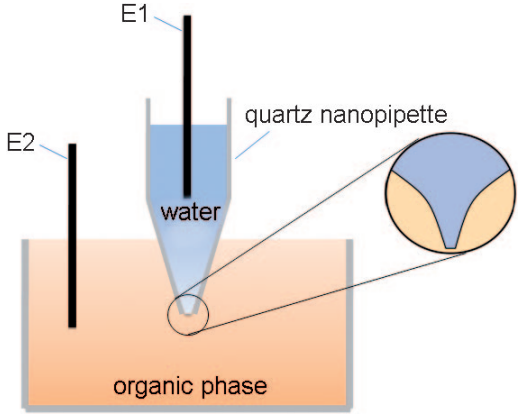

Figure 1. Schematic representation of the experimental setup. El and E2 are the reference electrodes in the aqueous and organic phases, respectively.

For an IT process at a micro-ITIES, the asymmetric diffusion pattern that results from the special geometry leads to an asymmetric cyclic voltammogram. However, the reasons for the pseudo steady-state response of IT reactions at a nanoITIES had hitherto not been elucidated. ${ }^{[5,7]}$ Figure 2 shows

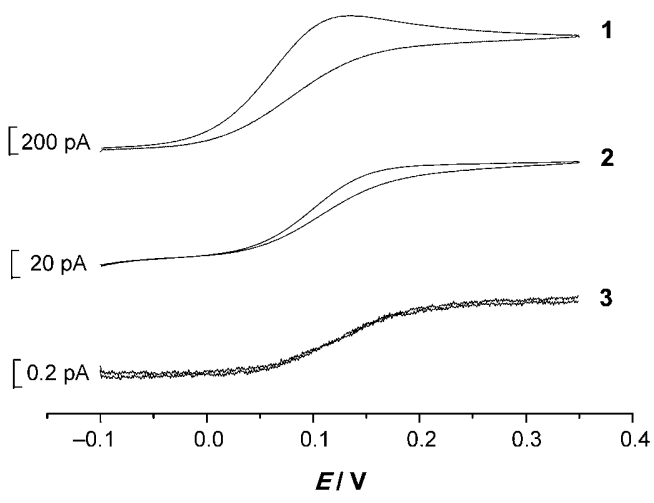

Figure 2. Cyclic voltammograms of TEA ${ }^{+}$transfer across the W/DCE interface supported at micro- and nanopipettes using Cell $A$. The radii of the pipettes are $4 \mu \mathrm{m}$ (curve 1), $100 \mathrm{~nm}$ (curve 2), and $1.2 \mathrm{~nm}$ (curve 3), respectively. Scan rate $=50 \mathrm{mVs}^{-1}$.

the cyclic voltammograms of $\mathrm{TEA}^{+}$transfer at the micro-and nano-W/DCE interfaces supported at the tip of pipettes. The shape of curve 1 (Figure 2) is asymmetric, in accordance with the asymmetric diffusion regime formed at the interface owing to the unique shape of the micropipette. Indeed during the forward scan, $\mathrm{TEA}^{+}$is driven out of the micropipette, creating a semilinear diffusion field, which results in a peakshaped current. On the reverse scan, the ingress transfer of $\mathrm{TEA}^{+}$is controlled by a hemispherical-type diffusion, which results in a steady-state current. The shape of the cyclic voltammogram changes significantly, and quasi steady-state cyclic voltammograms are obtained when the diameter of the pipette decreases from $\mu \mathrm{m}$ to nm (curves 2 and 3, Figure 2). The half-wave potential $\left(E_{1 / 2}\right)$ shift for $\mathrm{TEA}^{+}$transfer to more positive values as $r$ decreases is due to the change of the diffusion layer thickness ratio. ${ }^{[9]}$ The lack of a peak-shaped current response at a nano-ITIES was previously ascribed to the unique tip geometry that allows for steady-state nonlinear diffusion in the inner solution. ${ }^{[7]}$

To obtain a better understanding of this problem, two theoretical models have been here developed to calculate the current for an ion egress reaction at the nano-ITIES supported at nanopipettes (for more details see the Supporting Information). Equation (2) is the conclusion of the first

$I_{\mathrm{SS}} \approx \pi n F D c r \sin \theta$

model (Schwarz-Christoffel conformal mapping) describing the pipette shape with a hyperbola. Here $I_{\mathrm{SS}}$ is the steady-state current, $n$ the charge of the transferred ion, $F$ the Faraday constant, $D$ the diffusion coefficient, $c$ the bulk concentration of the egressing species, $r$ the inner pipette radius, and $\theta$ is the tip angle. Equation (3) sums up the second model in which the

$I_{\mathrm{SS}} \approx \pi n F D \operatorname{cr} \theta$

pipette is described as a perfect cone (solid-angle approach, see the Supporting Information). This model emphasizes that diffusion within the pipette is spherical and is focused towards the tip of the pipette. Because $\sin \theta \approx \theta$ when $\theta$ is small, the results of these two independent models converge for a typical $\theta$ value of $7^{\circ[10]}$ (deviation $\approx 0.2 \%$ ). Thus the current for an egress ion transfer process is indeed steady-state. Furthermore, the current is predicted to be proportional to the tip angle, and this is corroborated by the digital simulations by Amemiya et al. ${ }^{[9]}$ and Kakiuchi et al..$^{[10]}$

Here we compare Equations (2) and (3) with the empirical Equation (4) proposed by Beattie et al.,${ }^{[11]}$ which has been

$I_{\mathrm{SS}}=3.35 \pi n F D c r$

widely used to characterize the effective radii of micro- and nanopipettes using FIT of potassium by DB18C6 at a W/DCE interface. Here $c$ is the bulk concentration and $D$ the diffusion coefficient of the ionophore; the other parameters have the usual meanings. We investigated the egress transfer of TEA ${ }^{+}$ and FIT of $\mathrm{K}^{+}$by DB18C6 using identical sized nanopipettes $(r<5 \mathrm{~nm})$ and calculated the radii using Equations (3) and (4), respectively (see Table S1 in the Supporting Information). From this table, it is clear that the radius value calculated from Equation (3) is about three times larger than that obtained from Equation (4). This discrepancy is close to the difference between the steady-state current of nanopipettes with silanized outer walls $(\mathrm{RG} \approx 1.5)^{[12]}$ and those calculated by Equation (4) (2.3 times). This similarity implies that the nanoscopic interfaces involved may be approximately flat just like a disk electrode. This may be due to the interfacial tension increase as $r$ decreases, ${ }^{[13]}$ and the obvious evidence is that it is usually hard to fill the nanopipette in experiments.

Because the charging current is inherent at a nano-ITIES, a background subtraction has been used to obtain highquality voltammograms (Figure 3, see also Figures S3 and S4 in the Supporting Information). In previous IT kinetics experiments, the outer walls of the nanopipettes were usually silanized to eliminate the effect of possible leakage of the 


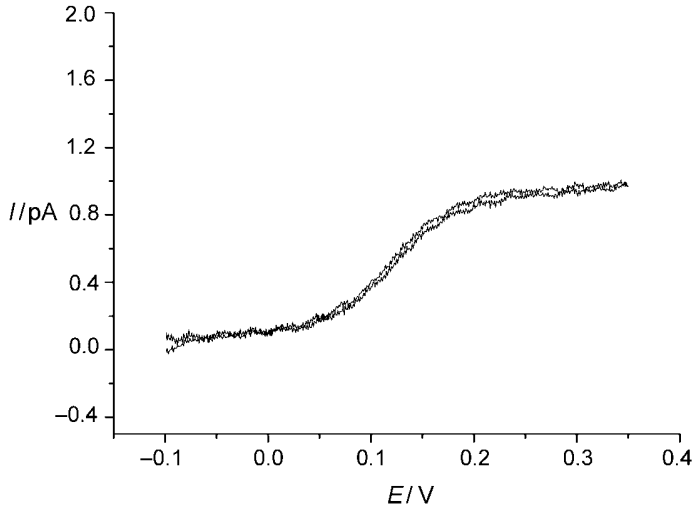

Figure 3. Background-subtracted cyclic voltammogram of $\mathrm{TEA}^{+}$transfer across the nano-W/DCE interface at the nanopipette $(r \approx 2.7 \mathrm{~nm})$ using Cell B. Scan rate $=50 \mathrm{mVs}^{-1}$.

aqueous solution. However, it is extraordinarily difficult to silanize the nanopipettes when the radii are smaller than $5 \mathrm{~nm}$ owing to the capillary phenomenon in the silanization process. $^{[3,5,7]}$ In addition, there is no significant effect of silanization on the evaluation of kinetics. ${ }^{[5,6]}$

The method used for extracting the kinetic parameters for ET in a heterogeneous reaction from the steady-state voltammogram of a quasi-reversible reaction is the threepoint method developed by Bard and Mirkin. ${ }^{[8]}$ Three parameters, namely the half-wave potential, $E_{1 / 2}$, and the quartile potentials $E_{1 / 4}$ and $E_{3 / 4}$, which can be obtained experimentally, are used to determine $k^{0}$ and $\alpha$. For a steadystate voltammogram at a uniformly accessible electrode, reliable kinetic data can be evaluated only if the following three criteria are satisfied: 1) $\left|\Delta E_{1 / 4}=E_{1 / 2}-E_{1 / 4}\right| \geq 30.5 \mathrm{mV}$, 2) $\left|\Delta E_{3 / 4}=E_{3 / 4}-E_{1 / 2}\right| \geq 31.0 \mathrm{mV}$, and 3) $\left|\Delta E_{3 / 4}\right| \geq\left|\Delta E_{1 / 4}\right|$, otherwise the reaction is reversible and no kinetic data can be obtained (see an example shown in Figure S3 in the Supporting Information).

Since the pioneering work of Mirkin et al.,${ }^{[3]}$ the values of $k^{0}$ reported have steadily increased. For example, $k^{0}=$ $2.6 \mathrm{~cm} \mathrm{~s}^{-1}$ (pipette radius $80 \mathrm{~nm}$ ) for $\mathrm{TEA}^{+}$transfer at $\mathrm{W} /$ DCE interface was reported. ${ }^{[5]}$ However, no study has been reported for IT processes with a pipette with radius less than $5 \mathrm{~nm}$. The key for the three-point method is to obtain a nice cyclic voltammogram. In order to do so, one needs to take care of shielding, background subtraction, fabrication of the reference electrode, cleanness of the pipette, purity of chemicals, and more importantly, improvements in the pulling programs (for details see the Supporting Information).

Using this approach, well-defined background-subtracted steady-state voltammograms for the transfers of $\mathrm{TEA}^{+}$ (Figure 3), $\mathrm{ClO}_{4}^{-}$(Figure S4a), and FIT of $\mathrm{K}^{+}$by DB18C6 (Figure S4b) could be obtained. Based on the analyses of these voltammograms, the kinetic parameters can be extracted and are listed in the respective Tables $1-3$. The mean values of $k^{0}$ and $\alpha$ of three IT reactions are $110 \pm 23$, $95 \pm 31,35 \pm 8 \mathrm{~cm} \mathrm{~s}^{-1}$ and $0.57 \pm 0.08,0.56 \pm 0.13,0.63 \pm 0.05$, respectively. These values are the highest rate constants reported so far for charge-transfer processes at an ITIES, and they corroborate early measurements with nanometer-sized
Table 1: Kinetic parameters for $\mathrm{TEA}^{+}$transfer across nano-W/DCE interfaces at nanopipettes of different radii. ${ }^{[a]}$

\begin{tabular}{lllll}
\hline$r[\mathrm{~nm}]$ & $\Delta E_{1 / 4}[\mathrm{mV}]$ & $\Delta E_{3 / 4}[\mathrm{mV}]$ & $A$ & $k^{0}\left[\mathrm{~cm} \mathrm{~s}^{-1}\right]$ \\
\hline 1.8 & 31.4 & 32.7 & 0.70 & 120 \\
1.5 & 37.6 & 43.2 & 0.49 & 77 \\
2.7 & 32.2 & 34.0 & 0.58 & 103 \\
1.2 & 34.8 & 37.4 & 0.60 & 108 \\
3.2 & 31.0 & 33.2 & 0.50 & 140 \\
& & & $0.57 \pm 0.08$ & $110 \pm 23$
\end{tabular}

[a] $D_{\text {TEA }^{+}}$in water $=1.2 \times 10^{-5} \mathrm{~cm}^{2} \mathrm{~s}^{-1} \cdot{ }^{[21]}$

Table 2: Kinetic parameters for $\mathrm{K}^{+}$transfer facilitated by DB $18 \mathrm{C} 6$ across the nano-W/DCE interfaces at nanopipettes of different radii. ${ }^{[a]}$

\begin{tabular}{lllll}
\hline$r[\mathrm{~nm}]$ & $\Delta E_{1 / 4}[\mathrm{mV}]$ & $\Delta E_{3 / 4}[\mathrm{mV}]$ & $\alpha$ & $k^{0}\left[\mathrm{~cm} \mathrm{~s}^{-1}\right]$ \\
\hline 1.4 & 31.2 & 32.8 & 0.50 & 139 \\
1.1 & 31.0 & 32.2 & 0.67 & 112 \\
1.3 & 33.1 & 36.0 & 0.52 & 83 \\
1.2 & 31.8 & 33.1 & 0.71 & 62 \\
2.0 & 32.4 & 37.2 & 0.38 & 79 \\
& & & $0.56 \pm 0.13$ & $95 \pm 31$
\end{tabular}

[a] $D_{\mathrm{DB} 18 \mathrm{C} 6}$ in $\mathrm{DCE}=5.2 \times 10^{-6} \mathrm{~cm}^{2} \mathrm{~s}^{-1} \cdot{ }^{3]}$

Table 3: Kinetic parameters for $\mathrm{ClO}_{4}{ }^{-}$transfer across nano-W/DCE interfaces at nanopipettes of different radii. ${ }^{[a]}$

\begin{tabular}{lllll}
\hline$r[\mathrm{~nm}]$ & $\Delta E_{1 / 4}[\mathrm{mV}]$ & $\Delta E_{3 / 4}[\mathrm{mV}]$ & $\alpha$ & $k^{0}\left[\mathrm{~cm} \mathrm{~s}^{-1}\right]$ \\
\hline 1.1 & 46.8 & 49.5 & 0.55 & 24 \\
1.0 & 41.7 & 46.0 & 0.61 & 32 \\
3.8 & 36.0 & 37.8 & 0.64 & 35 \\
2.4 & 35.8 & 37.7 & 0.67 & 45 \\
5.2 & 33.3 & 34.6 & 0.67 & 38 \\
& & & $0.63 \pm 0.05$ & $35 \pm 8$
\end{tabular}

[a] $D_{\mathrm{ClO}_{4}-}$ in water $=1.79 \times 10^{-5} \mathrm{~cm}^{2} \mathrm{~s}^{-1} \cdot{ }^{[22]}$

electrodes reported by Lewis et al. ${ }^{[14]}$ The systematic underestimation of $k^{0}$ in previous electrochemical measurements is probably a result of the inefficient mass transfer to the relatively large interface. For a pipette with a radius of $1 \mathrm{~nm}$, $m_{0}$ is $\geq 100 \mathrm{~cm} \mathrm{~s}^{-1}$ (assuming $D=10^{-5} \mathrm{~cm}^{2} \mathrm{~s}^{-1}$ ). Thus, the theoretical upper limit for the determinable IT rate constant for a heterogeneous reaction is increased to at least $500 \mathrm{~cm} \mathrm{~s}^{-1}$. ${ }^{[15]}$

The large measured values of the IT rate constant can be discussed within the frame of an interfacial diffusion model in which the standard rate constant can be defined as Equation (5), ${ }^{[16]}$ where $D_{\mathrm{i}}$ and $\Delta x$ are the diffusion coefficient

$k^{0}=\frac{D_{i}}{\Delta x}$

within the boundary layer and the thickness of that layer, respectively. The mechanism of simple IT of TEA ${ }^{+}$is different from that of FIT. In the case of a FIT with an excess of aqueous ions inside the pipette, it is a classical TIC/TID mechanism, ${ }^{[17]}$ and the thickness of the diffusion layer in the organic phase can be obtained from Equation (4). In the case of IT, the thickness of the diffusion layer in the aqueous phase 
of the egress reaction of $\mathrm{TEA}^{+}$can be deduced from Equation (3) (see the Supporting Information). Thus, the value of $\Delta x$ is in the range of a few angstroms to a few nanometers. Assuming values of $\Delta x \approx 1 \mathrm{~nm}$ and $D_{\text {i }}$ $\approx 10^{-5} \mathrm{~cm}^{2} \mathrm{~s}^{-1}$, one obtains $k^{0}=100 \mathrm{~cm} \mathrm{~s}^{-1}$, which is in good agreement with the values obtained in this work.

From these results it is clear that the rate constants of simple IT of TEA ${ }^{+}$and FIT of $\mathrm{K}^{+}$by DB18C6 are on the same order of magnitude. However, the anion transfer is about three times slower than cation transfer; this is similar to previous observations for FIT where facilitated anion-transfer reactions were shown to be slower than facilitated cationtransfer reactions. ${ }^{[18]}$ One possible reason is that anions are usually characterized by higher hydration energies $\left(\Delta G_{\text {hyd }}\right)$ than similarly charged and sized cations. ${ }^{[19]}$ Anions also generally have much more complex geometries. For instance, they can be spherical, linear, triangular or other patterns (the structure of $\mathrm{ClO}_{4}{ }^{-}$is tetrahedral). According to the Marcus theory for IT reactions, ${ }^{[20]}$ an IT reaction at an ITIES involves an initial desolvation from the first phase and then concerted solvation by the second phase. The higher the hydration energy, the harder it is to overcome this barrier. This thermodynamic "penalty" is likely to be reflected in slower anion-transfer kinetics. ${ }^{[18]}$

In summary, the kinetics of ion transfer at a W/DCE interface has been studied using nanopipette steady-state voltammetry, and the fastest kinetic data to date have been obtained. Two equations based on different theoretical models have been proposed to explain the steady-state current for ion egress reactions at the nano-ITIES supported at nanopipettes. This methodology offers advantages for its ultrafast mass transport rate and holds splendid promise for understanding the mechanism of IT processes at soft interfaces, chemical analysis, and biological sensing on the molecular scale.

\section{Experimental Section}

1,2-Dichloroethane (DCE), potassium chloride, lithium chloride, and sodium perchlorate were obtained from Beijing Chemical Co. Dibenzo[18]crown-6 (DB18C6, 98\%) was supplied by ACROS. Tetraethylammonium chloride (TEACl, 97\%), bis(triphenylphosphoranylidene)ammonium chloride (BTPPACl, $98 \%$ ), and potassium tetrakis(4-chlorophenyl)borate (KTPBCl, 98\%) were purchased from Fluka. All chemicals used were analytical grade or better. Water filtered through a Millipore filter was employed for to prepare the aqueous solutions. The DCE was washed several times with deionized water before use. Bis(triphenylphosphoranylidene)ammonium tetrakis(4-chlorophenyl) borate (BTPPATPBCl) was synthesized according to published procedures ${ }^{[7]}$ and used as the supporting electrolyte in the DCE phase.

Fabrication of nanopipettes: A Model P-2000 laser puller (Sutter Instrument) was used to prepare the nanopipettes with an orifice radius in the range of $1-5 \mathrm{~nm}$ from quartz capillaries $(1 \mathrm{~mm}$ outer diameter, $0.7 \mathrm{~mm}$ inner diameter). The tip radius and the length of the shank were controlled by the pulling parameters. The aqueous solution was filled from the back of the nanopipette using a $10 \mu \mathrm{L}$ syringe. The nanopipette was checked using an Olympus BX-51 optical microscope (BX-51, Olympus) prior to each measurement to ensure that no bubble trapped was inside.

Electrochemical measurements: Cyclic voltammograms were performed with a CHI 910B electrochemical workstation $(\mathrm{CH}$ Instruments, Inc.). An Ag wire (0.125 mm in diameter) coated with $\mathrm{AgCl}$ was inserted into the aqueous phase inside the pipette and used as the aqueous reference electrode. Another Ag wire coated with $\mathrm{AgTPBCl}$ was immersed in the outside organic phase and used as the organic reference electrode. All the experiments were carried out at room temperature $\left(22 \pm 2^{\circ} \mathrm{C}\right)$.

Received: June 11, 2009

Revised: July 21, 2009

Published online: September 18, 2009

Keywords: cyclic voltammetry · kinetics · interfaces · ion transfer . nanopipettes

[1] a) H. H. Girault in Modern Aspects of Electrochemistry, Vol. 25 (Eds.: J. O. M. Bockris, B. E. Conway, R. E. White), Plenum, New York, 1993, p. 1; b) A. G. Volkov, D. W. Deamer, LiquidLiquid Interfaces. Theory and Methods, CRC, Boca Raton, 1996; c) I. Benjamin, Chem. Rev. 1996, 96, 1449; d) F. Reymond, D. Fermin, H. Lee, H. H. Girault, Electrochim. Acta 2000, 45, 2647; e) B. Liu, M. V. Mirkin, Electroanalysis 2000, 12, 1433; f) P. Jing, S. He, Z. Liang, Y. Shao, Anal. Bioanal. Chem. 2006, 385, 428.

[2] G. Taylor, H. H. Girault, J. Electroanal. Chem. 1986, 208, 179.

[3] Y. Shao, M. V. Mirkin, J. Am. Chem. Soc. 1997, 119, 8103.

[4] G. Wittstock, M. Burchardt, S. E. Pust, Y. Shen, C. Zhao, Angew. Chem. 2007, 119, 1604; Angew. Chem. Int. Ed. 2007, 46, 1584.

[5] C. Cai, Y. Tong, M. V. Mirkin, J. Phys. Chem. B 2004, 108, 17872.

[6] Y. Yuan, Y. Shao, J. Phys. Chem. B 2002, 106, 7809.

[7] P. Jing, M. Zhang, H. Hu, X. Xu, Z. Liang, B. Li, L. Shen, S. Xie, C. Pereira, Y. Shao, Angew. Chem. 2006, 118, 7015; Angew. Chem. Int. Ed. 2006, 45, 6861.

[8] M. V. Mirkin, A. J. Bard, Anal. Chem. 1992, 64, 2293.

[9] P. J. Rodgers, S. Amemiya, Anal. Chem. 2007, 79, 9276.

[10] N. Nishi, S. Imakura, T. Kakiuchi, J. Electroanal. Chem. 2008, 621, 297.

[11] P. D. Beattie, A. Delay, H. Girault, J. Electroanal. Chem. 1995, 380, 167.

[12] Y. Shao, M. V. Mirkin, J. Phys. Chem. B 1998, 102, 9915.

[13] A. W. Adamson, A. P. Gast, Physical Chemistry of Surfaces, Wiley, New York, 1997.

[14] R. M. Penner, M. J. Heben, T. L. Longin, N. S. Lewis, Science 1990, 250, 1118 .

[15] P. Sun, M. V. Mirkin, Anal. Chem. 2006, 78, 6526.

[16] a) T. Kakiuchi, J. Electroanal. Chem. 1992, 322, 55; b) K. Kontturi, J. A. Manzanares, L. Murtomaki, D. J. Schiffrin, J. Phys. Chem. B 1997, 101, 10801.

[17] Y. Shao, M. D. Osborne, H. H. Girault, J. Electroanal. Chem. 1991, 318, 101.

[18] R. Cui, Q. Li, D. Gross, X. Meng, B. Li, M. Marquez, R. Yang, J. Sessler, Y. Shao, J. Am. Chem. Soc. 2008, 130, 14364.

[19] Y. Marcus, J. Chem. Soc. Faraday Trans. 1991, 87, 2995.

[20] R. A. Marcus, J. Chem. Phys. 2000, 113, 1618.

[21] P. Liljeroth, C. Johans, K. Kontturi, J. A. Manzamzres, J. Electroanal. Chem. 2000, 483, 37.

[22] D. R. Lide, Handbook of Chemistry and Physics, CRC, Boca Raton, FL, 76th ed., $\mathbf{1 9 9 5}$, p. 5. 